\title{
Noise and power programmability in semi-custom I/O buffers
}

Carro, L., Gonçalves, P.B., Kaiser, G. ,SuzimA.A.

Universidade Federal do Rio Grande do Sul

Departamento de Engenharia Elétrica

Av. Oswaldo Aranha 103

Porto Alegre - RS - BRASIL

CEP 90035-190

Fax: +55 512261171

Phone: +55513163516

e-mail: carro@iee.ufrgs.br

\begin{abstract}
This paper describes a new I/O buffer design, planed to allow low ringing at high frequencies at the presence of inductive package noise. Also, the buffer is configurable, so that the semi-custom designer can make a choice among power consumption, switching noise and delay. A reasonably simple and accurate model of an I/O buffer can be used to help semi-custom users foresee PAD behavior under different load and inductive packaging situations. Analysis results and simulations of the behavioral model were validated by a discrete prototype. The new buffer design was simulated, and reached higher frequencies without noise degradation when compared with traditional buffer design.
\end{abstract}

Keywords

Low power buffer design, low noise buffer design, high speed digital design, I/O buffer physical design 


\section{INTRODUCTION AND MOTIVATION}

During the design of a new Gate-Array matrix some developments were made regarding I/O buffers. Design trade-offs had to be made. Besides DC current capability ranging from 4 to $20 \mathrm{~mA}$, a special request regarding power consumption programmability had to be achieved, so that a designer could choose between a faster PAD or a low power consumption one. However, design should not loose its efficiency regarding speed. I/O circuitry concentrates large transistors, and hence large currents and output capacitances are to be expected. Also, a high number of output pins among the total number of PADs is very important, considering total power dissipation on the chip. The possibility of having programmable PADs regarding their power-delay product was so very desirable.

As the design proceeded, however, some parasitic effects like pin inductance showed that output capacitance was not the most important parameter, when one wants to reach medium to high frequencies. Not only power was affected, but the digital integrity of the output signal was threatened as well, caused by inductive noise. In order to maintain digital signal integrity, a new buffer architecture was developed, allowing fast switching speed but extremely low switching noise.

This work tries to summarize the design relations regarding the size of the output stage and a mathematical model able to help digital designers discover how to choose the correct compromise among PAD speed, PAD power, output load capacitance, parasitic inductance and digital signal integrity. To compensate ringing at power lines, a new circuit architecture is proposed and validated with simulations.

This paper is organized as follows: section 2 presents a study about power consumption in PADs, as a function of the delay and the parasitic power consumption. In section 3 the ground bounce problem is explained, and studied as a function of the switching speed of the PAD, the output capacitance, output transistor conductance and the parasitic inductance present in the chosen packaging. In section 4 a model able to help users foresee the behavior of the PAD is presented, and validated with experimental data. Section 5 presents the new PAD designed to cope with inductive noise, followed by simulation experiments. Finally, section 6 presents our conclusions.

\section{POWER AND PARASITIC POWER IN OUTPUT BUFFERS}

Total power dissipated in a system consisting of a pair buffer/load is divided between dynamic power (the one delivered to the load) and parasitic power, which is the one caused by the short-circuit current present when a certain input voltage causes both the output buffer transistors to be on. This current flowing between Vdd and Vss bypasses the load, and the duration of such charge displacement is a 
function of the rise and fall time of the input, which is in turn dependent on the relative gate sizes between 2 consecutive buffer stages.

Various studies are present in the literature ((Mead, 1980), (Veendrick, 1984), (Prunty,1992), (Choi 1994)), although some of them do not use realist output pad capacitances (above 50pF, for example). In order to study different trade-offs among power, delay and noise margins, various circuits were simulated with realistic capacitive loads. Using $\mathrm{CL}=50 \mathrm{pF}, \mathrm{f}=20 \mathrm{MHz}$ and $\mathrm{Vdd}=5$ Volts dynamic power reaches $25 \mathrm{~mW}$, exactly 10 times the power used as an example in (Veendrick, 1984).

Simulations with 1, 2, 3 and 4 stages buffers have been done, measuring power consumption, delay, rise and fall times. All buffers were simulated to $20 \mathrm{MHz}$ clock frequency, with a load capacitance of $50 \mathrm{pF}$. In each simulation the tapering factor was changed, and as a consequence the number of stages also changed. Table 1 shows the results of such study.

Table 1 - Buffers with 1,2,3 and 4 stages (simulation results)

\begin{tabular}{lllll} 
& one stage & two stages & three stages & four stages \\
\hline tap. fact. & 55.0 & 7.40 & 3.82 & 2.72 \\
Tot.Pow.(mW) & 33.6 & 29.3 & 30.1 & 31.8 \\
Din.Pow.(mW) & 25 & 25 & 25 & 25 \\
Par.Pow.(mW) & 8.6 & 4.3 & 5.1 & 6.8 \\
Delay(ns) & 7.25 & 3.49 & 3.26 & 3.39 \\
Trise/fall(ns) & 5.90 & 3.53 & 3.42 & 3.54 \\
Area(transist.) $\left(\mu \mathrm{m}^{2}\right)$ & 17.3 & 19.6 & 23.0 & 25.9 \\
\hline
\end{tabular}

Based on the results shown in table 1, we decided to adopt a 2 stage buffer. The conclusion of this first analysis is that, for circuits with realistic off-chip capacitive loads and frequencies, parasitic power reduction is not essential. Reduction in the number of stages allows area savings, since for a 4 stage buffers with $e$ as the tapering factor total area is $25.904 \mathrm{pm}^{2}$ in the used CMOS 1.2 technology, while a 2 stage buffer using 7.4 as the tapering factor reduces area to $19.584 \mathrm{pm}^{2}$, with a $24 \%$ reduction in area, to an increase of only $0.1 \mathrm{~ns}$ in the buffer delay. 


\section{THE PROBLEM OF PIN INDUCTANCE (GROUND-BOUNCE)}

The moment I/O circuits start to operate at higher frequencies, rise and fall times have to be shorter. Inevitably, the current variation over load capacitances in a given amount of time is also higher (di/dt). Output capacitances connected to an output PAD charge and discharge through VDD and VSS PADs. The di/dt caused by this charge movement will be translated to a voltage variation in the power supply pins of the circuit $\left(\mathrm{V}_{\mathrm{L}}=\mathrm{L} * \mathrm{di} / \mathrm{dt}\right)$, resulting in a phenomenon known as ground-bounce (Johnson, 1993).

According to the type of packaging used, different values of pin inductance will be present. For example, a 68 pin DIP presents $35 \mathrm{nH}$ inductance, while surface mount pins can have inductors as low as $7 \mathrm{nH}$ (Johnson, 1993). Practical measurements have shown inductance as high as $500 \mathrm{nH}$ for plastic pins packaging of the discrete transistors 4007 , for example.

Since the size of the output transistors has a determinant role in the groundbounce problem, initially we have fixed their size according to the available output PAD area. The first stage of an output PAD was taken as a minimum size inverter from the Gate-Array matrix. Size of the output transistors is an important variable to define the tapering factor between stages and the overall area of the output buffer. Other variables are load capacitance, rise and fall time and the switching frequency of the buffer.

To analyze noise problems originated by the ground-bounce we used a 2 stage buffer, adding I/O pin and power line inductors, as shown in Figure 1 (L1 to L9). We supposed the use of a power couple (Vdd/Vss) to every 4 output pins driving maximum current. This way, the discharge current (the one that flows from the capacitors to ground) and the charge currents (from Vdd to the output capacitor) is distributed among multiple power pins. Also, power pins to the core and to the PAD ring are assumed to be separated.

Initially, the circuit of Figure 1 was simulated with inductors L1 and L9 equal to $35 \mathrm{nH}$, and load capacitors of 50pF. X1 is an inverter build with basic transistors from the Gate -Array core, while X2 to X5 are two stage buffers as previously described.

In Figure 2 one can see the waveforms of the output voltage over C1. From top to bottom in this figure there are signals with frequencies of 5, 10 and $20 \mathrm{MHz}$ respectively. In the sequence, for the same set of frequencies, we have diminished the size of the output transistors by half. It can be verified that in the first three waveforms (the ones with larger transistors), there is a huge over-oscillation superposed to the output signal, caused by the combination of reactive inductance and capacitance at that pin. This superposed oscillation is favored by the high $\mathrm{Q}$ of the oscillating circuit, allowed by the small saturation resistance of the output buffer transistors. 


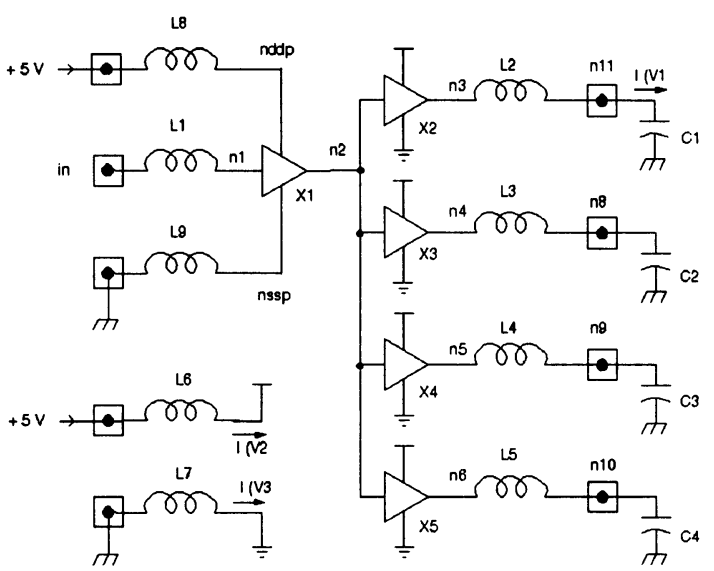

Figure 1 Simulated circuit

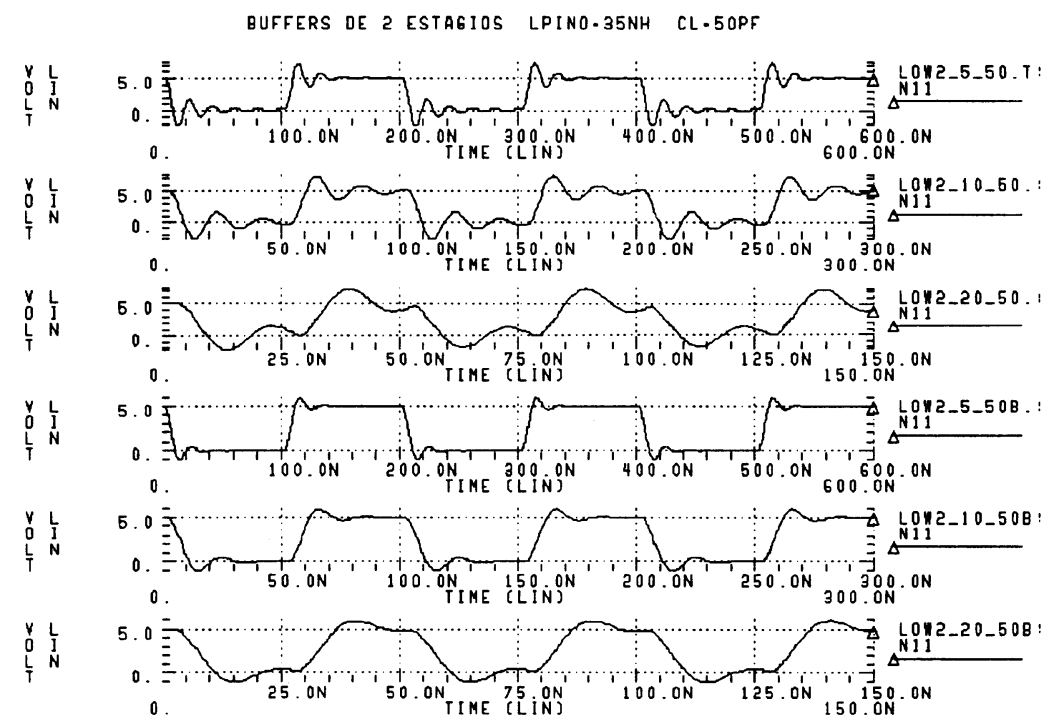

Figure 2 Voltage waveforms at $\mathrm{C} 1.1$ ) $\mathrm{f}=5 \mathrm{MHz} ; 2) \mathrm{f}=10 \mathrm{MHz} ; 3) \mathrm{f}=20 \mathrm{MHz}$;

$4,5,6)$ same frequencies, but half transistor sizes

Still referring to Figure 2, in waveforms 4 to 6 a meaningful reduction in the superposed oscillation has been obtained. This reduction is achieved thanks to the reduction in the $\mathrm{Q}$ factor by the increase of output resistance of the output transistors. Also, smaller output transistors allow smaller output currents at the power bus connections inside the chip. For large output transistors simulations showed a peak current of almost $50 \mathrm{~mA}$, a value too large when one has to think about many output PADs switching at the same time. For small output transistors only $130 \mathrm{~mA}$ were requested for a group of 4 PADs, against $200 \mathrm{~mA}$ in the case of 
large conductance transistors. At a frequency of $20 \mathrm{MHz}$ the output signal has a large amount of distortion, as shown in Figure 2. In Figure 3 one can see the output waveforms at $20 \mathrm{MHz}$, but with smaller parasitic inductors $(7 \mathrm{nH})$. The first and second waves refer to a $50 \mathrm{pF}$ load, while the other were simulated with a $100 \mathrm{pF}$ load. Waves one and three (top down) have larger output transistors, while the other have half the size of the other. In this case of low inductive packaging, buffers with half the maximum size can be used to charge loads up to $50 \mathrm{pF}$, while for $100 \mathrm{pF}$ or more the fastest transistor should be used, to avoid excessive output signal attenuation.

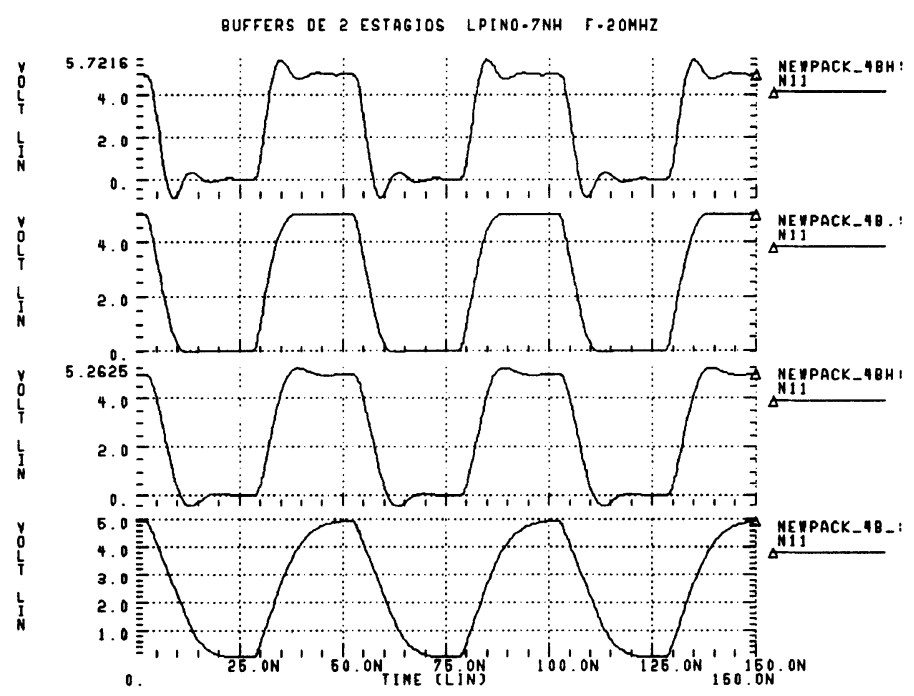

Figure 3 Output waveforms to $20 \mathrm{MHz}$ input, $7 \mathrm{nH}$ parasitic inductor. 1)Cl=50pF, $\left.\left.\left.\mathrm{W}_{\text {MAX }} ; 2\right) \mathrm{Cl}=50 \mathrm{pF}, \mathrm{W}_{\text {MAX }} / 2 ; 3\right) \mathrm{Cl}=100 \mathrm{pF}, \mathrm{W}_{\text {MAX }} ; 4\right) \mathrm{Cl}=100 \mathrm{pF}, \mathrm{W}_{\text {MAX }} / 2$;

The analysis of simulation results allows the formulation of a design strategy at hand for the semi-custom user engineer. In order to diminish the amount of ground-bounce, one possible solution is to divide the output transistor in 2 smaller ones. By metalization the designer can choose between a faster PAD to highly capacitive loads or to a slower PAD to small capacitances with less danger of signal ringing. At the same load conditions, large transistors allow faster rise and fall times, at the price of a higher ground-bounce when high inductance packaging is used. In this last case, a series termination must be added to cancel out the parasitic oscillations. Using the smaller transistor pair will cause a degradation of nominal performance (delay, rise and fall time), but a safer circuit regarding oscillations. 


\section{BEHAVIORAL MODEL}

Since physical parameters like parasitic inductance and load capacitance can not be changed, the final decision of which PAD circuit to use (more resistive and slower, more conductive and more oscillation prone) must be taken by the final GateArray user, or by a CAD tool. In both cases a behavioral model is necessary, taking all design parameters into account and giving reliable results regarding the final circuit behavior under the desired load.

A very simple second order model has been developed, shown in Figure 4. As in Figure 1, GND inductors present at the GND pin are also represented. The output transistor was substituted by a voltage source and series resistor, representing the output high to low transition and the output transistor resistance when in saturation. Only the high-low transition was taken into account, for the low-high one should be similar by assuming proper transistor sizing to assure equal noise margins.

The model shown in Figure 4 has the advantages of simplicity and easiness of use, being rightly manipulated by the final user or the CAD system. The substitution of the transistor by a voltage source and a resistor might cause amplitude errors, for the transistor will move from the saturation to the linear region, increasing the time it takes to discharge the capacitance. These errors, however, should be small, given the large amplitude of the oscillations at the beginning of the transition, when the transistor is saturated.

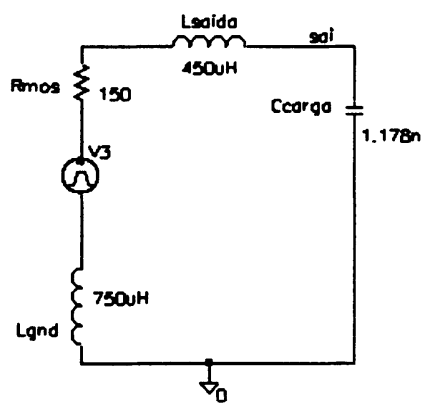

Figure 4 Second order model representing a PAD

In order to develop the model we just verified that there are only two energy storing elements. Since it is much easier to work at the frequency domain in order to solve the differential equations present ate the circuit, we just compute the answer at the s-plane and applied the inverse transform. The only approximation was to consider a linear resistor, which is not critical, as already mentioned.

The output voltage over the load $\left(V_{o u T}\right)$ can be expressed in the s-plane as the response of the transfer function to a voltage step, and one writes 
$\operatorname{Vout}(s)=\frac{\omega}{(s+a)^{2}+\omega^{2}}$

This equation has a time domain equivalent given by

$\operatorname{Vout}(t)=e^{-a t} \cdot \sin (\omega t)$.

Equation (2) means that the response of the circuit is a sinusoid function, exponentially attenuated. This result is easily verified in Figures 2 and 3. The term $\omega$ in equations (1) and (2) is the oscillation radian frequency, given by the parasitic inductor and the load, $1 / \mathrm{SQRT}\left(\mathrm{L}^{*} \mathrm{C}\right)$. The term $\boldsymbol{a}$ is the attenuation factor, given by L/R. Again, this is coherent with Figures 2 and 3, where the increase in the output resistance or the decrease in packaging inductance greatly diminished oscillations.

To validate the model presented in Figure 4 a circuit using discrete 4007 devices has been used. In order to carry the analysis in a meaningful way, we first developed a frequency scaling, so that all inductors and capacitors had their values multiplied by 1000 , while the frequency was reduced by the same factor. This way, parasitic inductors and capacitors present in the 4007 packaging would not be meaningful to the desired measurement, in comparison with the relative large inductor placed outside the chip. The actual conductance of output devices of a given technology is not important, when one wants to compute the oscillation frequency. What really matters is the relative size of the series impedances on frequency, so that the exponential envelope attenuates the oscillations.

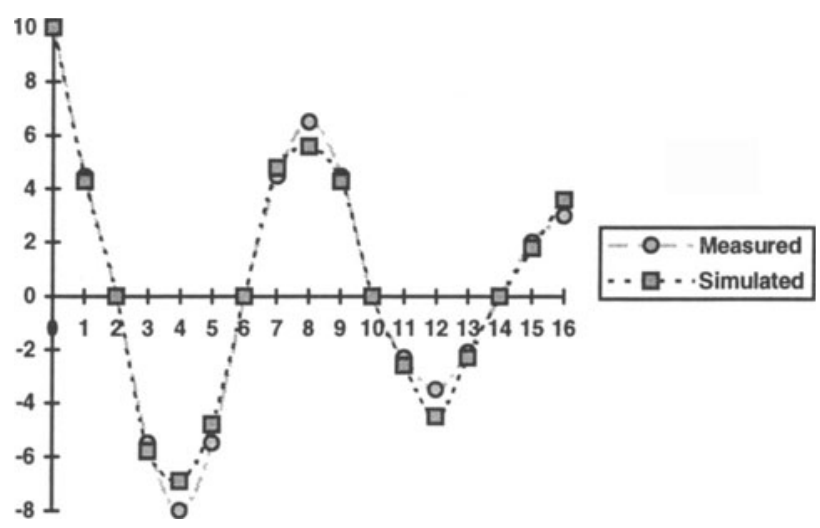

Figure 5 Comparison of theoretical and experimental data 
Experimental results, shown in Figure 5, show good agreement with the proposed model. The difference between the proposed equation and reality was less than $9 \%$, only when the amplitude of oscillations were small, caused by the change in transistor conductance when moving from saturation to the linear region.

Another solution like a strobe signal to precharge the output to $\mathrm{Vdd} / 2$ before the switching occurs could be used (Kang, 1996). In this case, the amount of needed current is smaller, for the output is already at half the final value. Nevertheless, this type of solution has 2 drawbacks: the need of an internally generated strobe signal, highly timing dependent and tricky for the system designer to cope with. Also, total power would increase, for to maintain the output at half Vdd there must be a path from the power line to GND.

\section{NEW PAD DESIGN}

Although the semi-custom engineer can choose between a faster or more conservative PAD, the ideal situation would be the availability of a fast buffer without inductive noise. The goal is under research among different laboratories, and some results have been achieved, mainly for what regards transmission line terminations ((Sechler,1995), (Johnson, 1993)). There is not available, to the authors knowledge, a circuit able to cope with inductive noise, present at low cost packaging at medium to high speed designs.

In order to solve the problem we considered the reasoning behind the proposed model. Since a resistive circuit greatly attenuates the noise, but slows down circuit performance, what one needs is a fast transistor that will allow fast transitions, and gradually this fast transistor should turn into a large resistor, increasing the attenuation factor and avoiding parasitic oscillations.

One of the ways to achieve this goal was to use a self-timed buffer, that could turn itself off after the output signal had reached the desired voltage level. Instead of using self-timed logic, we relied on the analog nature of transistors. Figure 6 shows the new proposed architecture (the n-part of the circuit, the p-part is just its dual). The main idea is the following: the new PAD should be fast enough to rise the output voltage up to Vt (threshold voltage) of the next stage, so as to start a new transition. From this point on, the PAD should immediately increase its resistance, so that all oscillations would disappear.

The new PAD was developed following the proposed philosophy. The output transistor was split into two (MNinv and M1), with a ratio of 4:1, the larger transistor being M1. As it is shown in Figure 6, at the moment the input signal Vin is at logic 1, the output should be discharged. At the beginning of the transition, M3 is conducting, while M2 is off. Both M1 and MNinv are on, so the PAD is working with maximum conductance. As Vout goes down, M3 starts to cut-off, at the same time that M2 turns on. Since M2 will remove charges from the gate of M1, M1 will increase its resistance, avoiding ringing. 


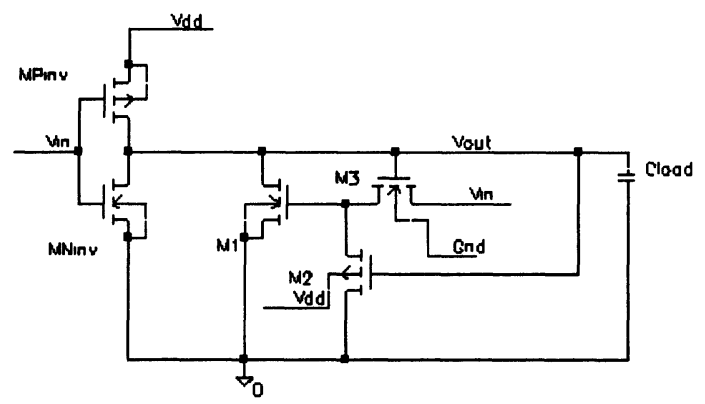

Figure 6 New PAD architecture

After the transition phase M3 will be completely off, and M2 on. Since Vds of M2 is equal to Vgs of M1, and Vds of M2 can not be smaller than Vtp, M1 will always be at a small conduction value, but at the linear region, not in saturation, with a larger resistance. When a low to high transition at the output node is needed, it should be noticed that although M1 will be conducting until Vout reaches Vtp, the amount of lost power caused by parasitic currents is the same as in the normal output buffers, when both $\mathrm{p}$ and $\mathrm{n}$ transistors are on.

Figure 7 presents simulation results of the proposed circuit, in a $1.2 \mathrm{CMOS}$ technology, with parasitic inductors of $35 \mathrm{nH}$ and load capacitor of $100 \mathrm{pF}$, at 33 $\mathrm{MHz}$, considering a short transmission line connecting 2 chips. The output signal is taken after an hypothetical input inverter of the second chip. It should be noticed that the normal I/O buffer does not work any more (signal outln), for the next stage has some spurious transitions. On the other hand, the compensated buffer (signal outl) works fairly well, allowing the system to reach such frequencies. At low to medium frequencies, ringing problem is not so evident (di/dt is small), and the compensated buffer causes a delay of 1 to 2 nanoseconds.

\section{CONCLUSIONS}

The design of a low power PAD as proposed in (Veendrick, 1984) could not be achieved, since parasitic short-circuit current power savings are not meaningful when realistic output loads and frequencies are used, like $50 \mathrm{pF}$ and 10 to $20 \mathrm{MHz}$.

The design of an output buffer must take into account trade-offs between power dissipation and delay, besides rise and fall times. The choice for a faster PAD might not be the right one, because this can cause severe output oscillations when small loads are used. In this case, either the user slows down the system (in order to wait it to stabilize) or a series termination must be used to increase output resistance so as to attenuate the undesired oscillations. 


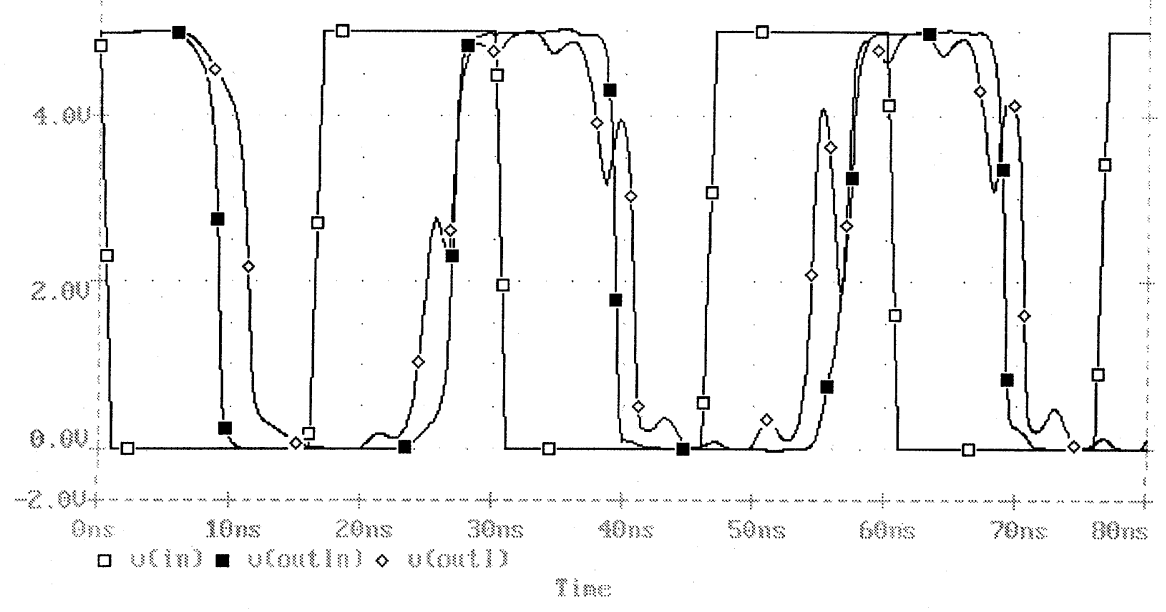

Figure 7 Simulation results of the new PAD: $\mathrm{L}=35 \mathrm{nH}, \mathrm{C}=75 \mathrm{pF}$

The trade-off between noise margins and speed is responsibility of the GateArray user, or system designer, taking into account the output load capacitance and the packaging inductance. In order to help the designer or to give data the supporting CAD, a simple second order model was proposed and validated, being quite effective to foresee circuit behavior. In the case when a fast PAD is chosen to drive small loads, severe oscillations might be present, and must be attenuated by a series resistor termination, that will affect rise and fall times.

Alternatively, we proposed a new PAD architecture, with varying resistance, able to cope with fast transitions and allowing small inductive noise. The proposed PAD has reached higher frequencies than the traditional one, thanks to the elimination of ground bounce by the use of a variable conductance transistor.

\section{REFERENCES}

Bellaouar, A.; El,asry, M. (1995) Low Power Digital VLSI Design-Circuits and Systems. Kluwer Academic Press, Boston.

Choi, J.-S.; Lee, K. (1994) Design of CMOS Tapered Buffer for Minimum PowerDelay Product, IEEE Journal of Solid-State Circuits, vol.SC-29, no.9, Sep. Johnson, H.; Graham, M. (1993) High Speed Digital Design. Prentice Hall, p.447.

Kang, S.; Leblebici, Y. (1996) CMOS Digital Integrated Circuits: Analysis and Design, Chapter 12. McGraw-Hill, New York. p.614

Mead, C.; Conway, L., (1980) Introduction to VLSI Systems, Addison-Wesley, Massachussets.

Prunty, C.; Gal, L. (1992) Optimum Tapered Buffer, IEEE Journal of Solid-State Circuits, vol.SC-27, no.1, Jan. 
Sechler, R. F. (1995) Interconnect design with VLSI CMOS. IBM Journal of Research and Development, v.39, n1/2, p.23-42.

Veendrick, H. (1984) Short-Circuit Dissipation of Static CMOS Circuitry and its Impact on the Design of Buffer Circuits, IEEE Journal of Solid-State Circuits, vol.SC-19, n.4, Aug.

\section{BIOGRAPHY}

Luigi Carro was born in Porto Alegre, Brasil, on Sept. 8, 1962. He received the electrical engineer degree from the Federal University of Rio Grande do Sul (UFRGS) in 1985, M.S. and Ph.D. degrees in Computer Science in 1989 and 1996, respectively. He was at ST-Microelectronics R\&D center, Agrate, Italia, from 1989-1991. Mr. Carro is a lecturer at the Electrical Engineering department, UFRGS. Areas of interest: VLSI processors and synthesis of complex systems.

Pedro Benoni Santos Gonçalves was born in Porto Alegre, Brasil, on May 27, 1969. He is an undergraduate electronic engineering student at UFRGS since 1994, being undergraduate researcher in the areas on $\mathrm{CAD}$ analog circuit design.

Altamiro Amadeu Suzim was born in Rio Grande do Sul, Brasil, Nov. 23, 1945. Electronic Engineer, UFRGS, 1972. MS Computer Science, UFRGS, 1977. DEA from Institut National Politechnique (INPG), Grenoble, France in 1979. Docteur Engineur degree from INPG-Grenoble 1981. Mr. Suzim has pos-doctoral studies at INPG, 1985, and Catholic University of Louvain-la-Neuve, 1988, Belgium. He is a full professor at the Electrical Engineering and Computer Science Department. He was also a professor of Post-graduation course in Industrial Computer Science, CEFET, Parana, from 1987-1988, and vice-president of SBmicro (from 1992 to 1994). Mr. Suzim is an ad-hoc consultant to CNPq, Finep and Fapergs. Areas of interest: VLSI processors.

Gustavo Kaiser was born in Porto Alegre, Rio Grande do Sul, Brasil, on December 20/1961. He received the electronic engineer degree from PUCRS in 1986, and the M.S. Computer Science degree from UFRGS in 1995. He is presently at the CRT-Telephone Company. Areas of interest: data communication, VLSI circuits. 